NEW NIST PUBLICATION

August 1989

\section{TRIAL OF OPEN SYSTEMS INTERCONNECTION (OSI) PROTOCOLS OVER INTEGRATED SERVICES DIGITAL NETWORK (ISDN)}

\section{Carol A. Edgar}

U.S. DEPARTMENT OF COMMERCE National Institute of Standards and Tochnology National Computer Systems Laboratory Gaithorsburg, MD 20899 



\section{TRIAL OF OPEN SYSTEMS INTERCONNECTION (OSI) PROTOCOLS OVER INTEGRATED SERVICES DIGITAL NETWORK (ISDN)}

\section{Carol A. Edgar}

U.S. DEPARTMENT OF COMMERCE National Institute of Standards and Technology National Computer Systems Laboratory Galthersburg, MD 20899

August 1989

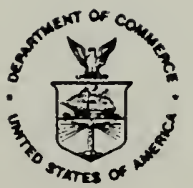

U.S. DEPARTMENT OF COMMERCE Robert A. Mosbacher, Secretary NATIONAL INSTITUTE OF STANDARDS AND TECHNOLOGY Raymond G. Kammer, Acting Director 



\section{SCLA IMER}

Certain commercial equipment, instruments, or materials are identified in this report in order to adequately specify the experimental procedure. Such identification does not imply recommendation or endorsement by the National Institute of Standards and Technology, nor does it imply that the materials or equipment identified are necessarily the best available for the purpose. 


\section{TABLE OF CONTENTS}

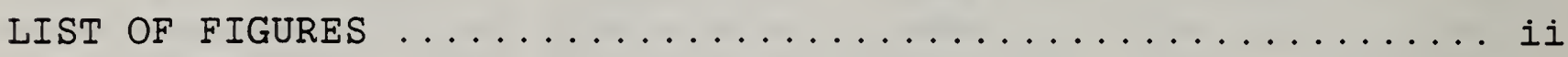

1.0 Introduction to the Trial .................... I

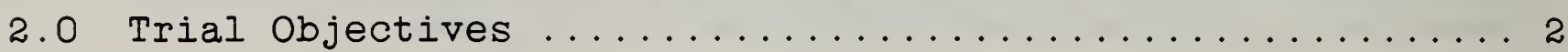

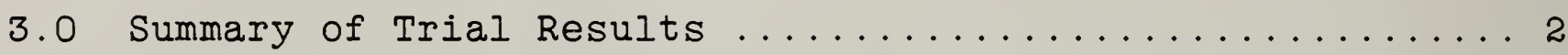

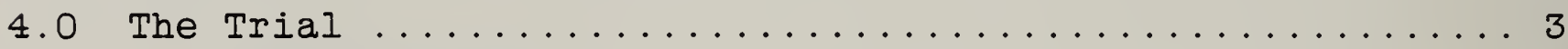

4.1 The Trial Topology ..................... 3

4.2 The Trial Configuration ................. 4

4.3 The Trial Parameters ................. 8

4.4 Additions to the Trial Configuration ............ 9

5.0 The Trial Results $\ldots \ldots \ldots \ldots \ldots \ldots \ldots \ldots \ldots \ldots$

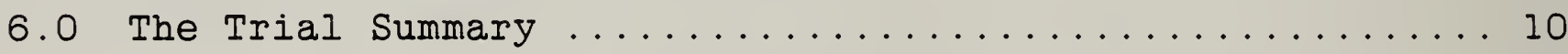

6.1 ISDN Functions Proposed in GOSIP Version $2 \ldots \ldots \ldots 10$

6.2 ISDN Functions Demonstrated During The Trial ...... 10

6.3 ISDN Functions Not Demonstrated During The

Trial ......................... 11

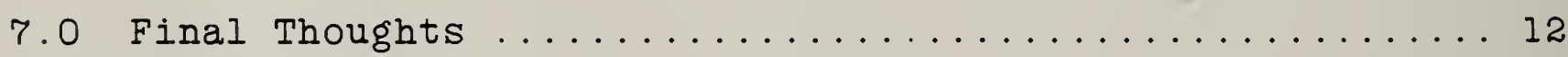

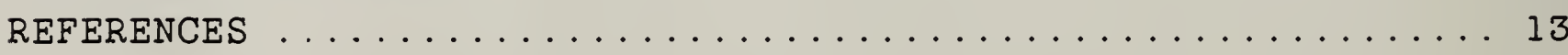

APPENDIX A - List of Contacts .................. 14 


\section{LIST OF FIGURES}

Figure 1 - OSI/ISDN Trial Configuration ............. 5

Figure 2 - OSI/ISDN Trial - End System Connectivity ....... 6 



\subsection{Introduction to the Trial}

This report contains the results of a trial demonstration of Open Systems Interconnection (OSI) protocols over Integrated Services Digital Network (ISDN) Trial. The trial was organized by NIST to demonstrate the use of ISDN as a lower layer technology for OSI applications. This trial was a necessary step for including ISDN as a lower layer technology in the GOSIP Version 2.

In 1988, the Government Open Systems Interconnection Profile (GOSIP) was released as FIPS 146. GOSIP addresses communication and interoperation between end systems and intermediate systems. It provides specific peer-level, process-to-process, and terminal access functionality between computer system users within and across government agencies.

The GOSIP specifies a set of OSI protocols for computer networking that is intended for use by government agencies. Starting in August 1990, the GOSIP protocols should be used by all Federal Government agencies in procurement of products and services which provide equivalent functionality to the OSI protocols referenced in FIPS 146 .

The GOSIP Version 1 supports the Message Handling Systems (MHS) and File Transfer, Access, and Management (FTAM) applications. It also supports interconnection of the following technologies:

* CCITT Recommendation X.25

* Carrier Sense Multiple Access with Collision Detection (IEEE 802.3)

* Token Bus (IEEE 802.4)

* Token Ring (IEEE 802.5)

The GOSIP Version 2 proposes additional functionality not included in the GOSIP Version 1 . The following additional functionality is being proposed for inclusion in the GOSIP Version 2:

* Virtual Terminal Service (TELNET and Forms profiles)

* Office Document Architecture

* Integrated Services Digital Network

* End System - Intermediate System protocol

* Connectionless Transport Service

* Connection Oriented Network Service

The GOSIP Version 2 is currently being released for public comment. This report addresses the applicability and feasibility of including ISDN as a lower layer technology in Version 2 of the GOSIP. 
The trial objectives, a summary of the trial results, the description of the trial configuration, and results obtained from its operation are discussed below.

\subsection{Trial Objectives}

The trial had three major objectives. The first objective, mentioned above, was to support the inclusion of ISDN transport capabilities in the GOSIP Version 2.

Demonstrating the operation of OSI applications using ISDN transport capabilities was the second objective. The trial was designed to prove that ISDN is a feasible alternative OSI transport medium. The design was specifically aimed to demonstrate that OSI end systems can also operate as native ISDN terminal equipment (with integrated ISDN capability rather than via a terminal adapter).

Finally, the trial demonstrated the interworking of ISDN and existing transport technologies. The operation of OSI end systems across a concatenation of subnetworks that include ISDN was demonstrated. This included the case where one of the OSI end systems is directly connected to the ISDN. Additionally, the case where no OSI end system is directly connected to ISDN was also demonstrated. That is, the ISDN serves as a transit subnetwork to connect two subnetworks (e.8., LANs) geographically separated.

\subsection{Summary of Trial Results}

The trial results demonstrate that the mandatory GOSIP protocols, Transport Class 4 (TP4), and the Connectionless Network Protocol (CLNP) can use ISDN bearer services to transport GOSIP application information for electronic mail ( $\mathrm{X} .400$ ) and file transfer (FTAM). The trial results also demonstrate that GOSIP-compliant end systems and intermediate systems can attach directly to ISDN services and that ISDN services can be used as a transit network, connecting local area networks and $X .25$ public data networks via CLNP routing devices. Finally, the trial demonstrated that the GOSIP protocol stack (i.e., Transport Class O (TPO) and Connection Oriented Network Service (CONS)) required for interconnecting to public electronic mail services can operate using ISDN services.

While the trial did not demonstrate all ISDN features proposed within Version 2 of GOSIP, about $70 \%$ of the proposed functions were demonstrated. The testing of dynamic B-channel allocation 
made directly through OSI software was very limited. Interconnection of computers to the ISDN service using the Primary Rate Interface (PRI) was not demonstrated.

The trial demonstrated the technical feasibility of including ISDN services into GOSIP Version 2 . The trial did not address issues of widespread interoperability between terminal equipment manufacturers and a variety of ISDN switch providers, although this is an objective of the GOSIP specification. The trial also did not address issues of interoperability between ISDN switches from a variety of vendors; this issue, while important to all users of ISDN services, is outside the scope of the GOSIP specification.

The trial, a collaboration between Government and industry, was a success. Many technical capabilities were evaluated in a short period. Continued Government and industry collaboration is required to guide the development of interoperable OSI and ISDN toward widespread utility and acceptance.

\subsection{The Trial}

Within the next several paragraphs the topology and configuration of the trial are discussed. Also discussed are the participants of the trial and the hardware/software that each provided. The discussion of the trial configuration will include such items as paths to be tested and parameter settings used.

\subsection{The Trial Topology}

The main component of the trial topology was an already-installed 5ESS (TM). The symbol (TM) signifies Trade Mark throughout this document. The 5ESS (TM) provided the ISDN capabilities. Attached to the 5ESS (TM) were OSI end systems operating OSI applications. The OSI applications demonstrated were the File Transfer, Access, and Management (FTAM) and the X.400 Message Handing System (MHS). These protocols were chosen for their maturity based on the availability of implementation agreements and vendor product offerings.

The tests executed during the interoperability testing were tests from the OSINET interoperability test suites. The correct titles for the documents are listed below:

* OSINET/EurOSInet X.400 Interoperability Tests, Version 1.00 
* OSIone FTAM Interoperability Tests, June 1989

Each of these documents is available from the NIST, from Carol Edgar at (301) 975-3613.

The ISDN capabilities provided by the 5ESS (TM) were interconnected with subnetworks of a different technology to show the interworking of ISDN with existing technologies. Specifically, the ISDN was interconnected with several IEEE 802.3 Local Area Networks (LANs) via OSI intermediate systems to allow end systems to communicate across the concatenation of several subnetworks. Connectivity to OSINET and an IEEE 802.4 LAN was also demonstrated. Figure 1 indicates the topology of the trial.

ISDN connectivity between the 5ESS (TM) and OSI end systems was provided via BRI interfaces. Those interfaces were either via a D-Channel or via a preallocated (or nailed-up) B-Channel. Figure 2 lists the trial path, end system path, the ISDN connectivity associated with that path, and the application tested.

The trial was made possible due to the efforts and participation of several organizations. Each of the participants and the hardware/ software they provided are noted on Figure 1. A list of contacts for each participating organization is provided in Appendiz A for detailed system inquiry.

\subsection{The Trial Configuration}

This section will step through the different paths provided by the trial configuration. Figures 1 and 2 are useful references in working through this section.

The trial configuration consisted of five main communication paths. Path 1 involved the NIST OSI workstation (ESA) exchanging electronic mail or files with the OSI workstation (ESC) provided by Mather Air Force Base. The hardware view of this path consisted of two Intermediate systems (ISS, ISI and IS2) directly connected to the 5ESS (TM) Switch via native BRI interfaces. Each IS was connected to an IEEE 802.3 LAN via a native controller board. Directly connected to each LAN was an End System (ES).

The software involved in this path consisted of OSI $\mathbb{X} .400$ MHS and FTAM implementations as part of an OSI stack. The remainder of the OSI stack consisted of Transport Class 4 (TP4) and the Protocol for the provision of the Connectionless Network Service (CLNP). The ISs were operating software appropriate to CLNP datagram 


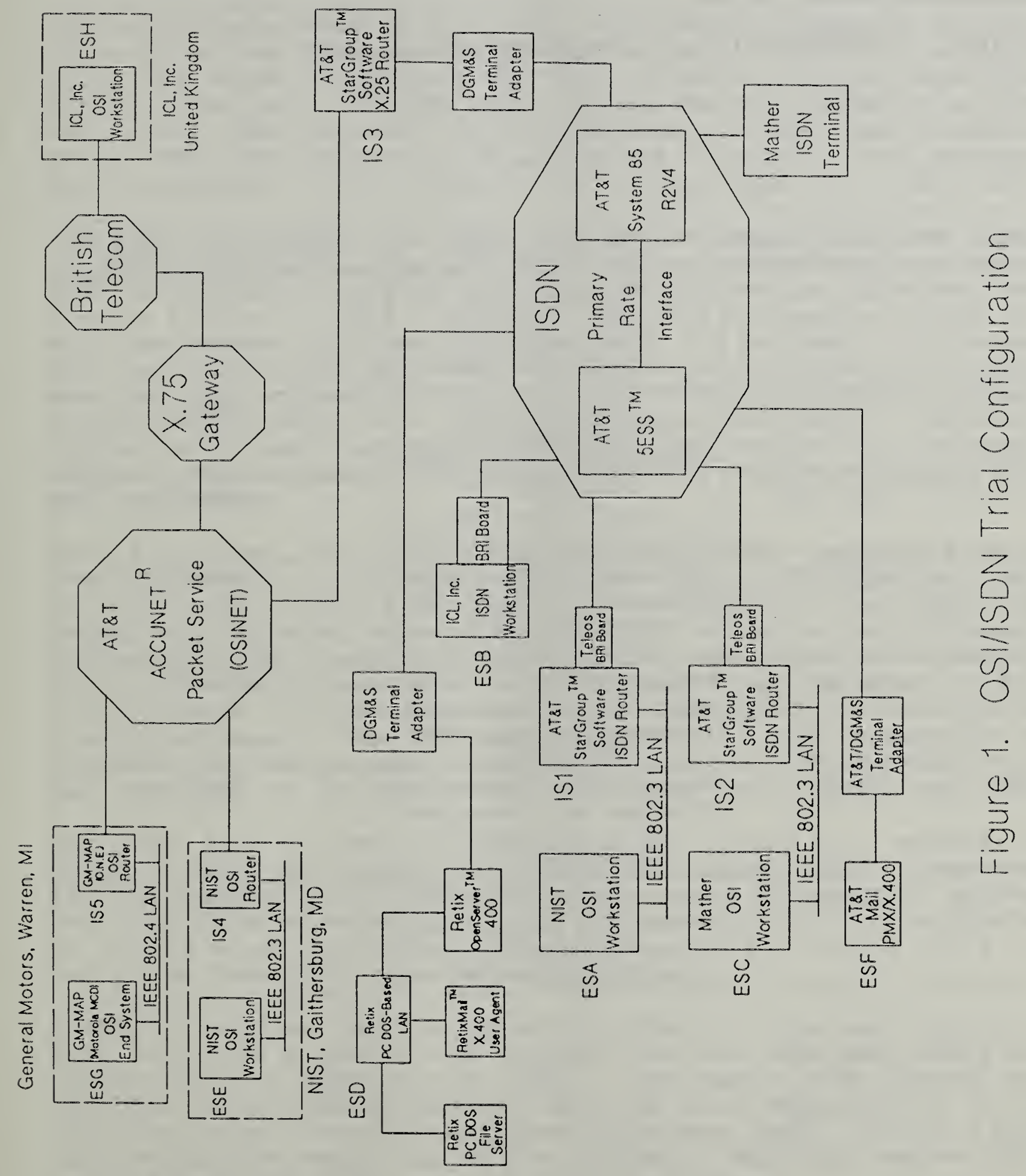


Figure 2. OSI/ISDN Trial - End System Connectivity

\begin{tabular}{|c|c|c|c|}
\hline Path & End System & ISDN Connectivity & Application Tested \\
\hline 1 & ESA - ESC & $\begin{array}{l}\text { ESA via } 802.3 \text { to IS1 } \\
\text { IS1 via D-Channel to ISDN } \\
\text { ESC via } 802.3 \text { to IS2 } \\
\text { IS2 via D-Channel to ISDN }\end{array}$ & FTAM \\
\hline 2 & $E S A-E S B$ & $\begin{array}{c}\text { ESA via } 802.3 \text { to IS1 } \\
\text { ISI via D-Channel to ISDN } \\
\text { ESB via nailed-up B-Channel to ISON }\end{array}$ & FTAM \\
\hline 3 & $E S A-E S D$ & $\begin{array}{c}\text { ESA via } 802.3 \text { to IS1 } \\
\text { IS1 via D-Channel to ISDN } \\
\text { ESD via nailed-up B-Channel to ISON }\end{array}$ & X.400 MHS \\
\hline 4 & $E S B-E S E$ & $\begin{array}{c}\text { ESB via nailed-up B-Channel to ISDN } \\
\text { IS3 via nailed-up B-Channel to ISDN } \\
\text { IS } 4 \text { via X.25 to OSINET } \\
\text { ESE via } 802.3 \text { to IS4 }\end{array}$ & FTAM \\
\hline 5 & $E S D-E S F$ & $\begin{array}{l}\text { ESD via nailed-up B-Channel to ISDN } \\
\text { ESF via nailed-up B-Channel to ISDN } \\
\text { * modification of this using a remote } \\
\text { ISDN terminal }\end{array}$ & $X .400 \mathrm{MHS}$ \\
\hline 6 & $E S B-E S G$ & $\begin{array}{l}\text { ESB via nailed-up B-Channel to ISDN } \\
\text { IS3 via nailed-up B-Channel to ISDN } \\
\text { IS5 via X.25 to OSINET } \\
\text { ESG via } 802.4 \text { to IS5 }\end{array}$ & FTAM \\
\hline 7 & $E S B-E S H$ & $\begin{array}{c}\text { ESB via nailed-up B-Channel to ISDN } \\
\text { IS3 via nailed-up B-Channel to ISDN } \\
\text { ESH via X.25 to British Telecom } \\
\text { via X.75 Gateway to OSINET }\end{array}$ & FTAM \\
\hline
\end{tabular}


routers (data packets between the ESS were routed through the ISs).

The second path found in the trial configuration required the NIST OSI workstation (ESA) and the ICL ISDN workstation (ESB) to exchange files with each other using OSI FTAM software. The hardware view differs from path 1 in that only 1 of the ESs is located on an IEEE 802.3 LAN. The other ES, the ICL workstation (ESB), was connected to the 5ESS (TM) switch via a native BRI interface. The software view consisted of OSI stacks containing FTAM, TP4, and CLNP.

The NIST OSI workstation (ESA) and the RetixMail (TM) User Agent and Servers (collectively ESD) were required to exchange electronic mail in the third path of the trial configuration. The hardware view is similar to that of path 2. The only difference is that the Retix end system (ESD) actually consisted of a RetizMail (TM) Server with several Clients attached to a LAN. It is important to note, that for the purpose of this trial the RetixMail (TM) Server and Clients were viewed as a single distributed ES (ESD). The Retix system (ESD) was connected to the 5ESS (TM) switch via a Terminal Adapter. The software view consisted of OSI stacks containing MHS, TP4, and CLNP.

Path 4 involved the ICL ISDN workstation (ESB) exchanging files over OSINET with a NIST OSI workstation (ESE) in Gaithersburg, MD. In this step, a third IS (IS3) was connected to the 5ESS (TM) switch via a Terminal Adapter and to OSINET via a native $\mathbf{Z} .25$ board. Another IS (IS4, located in Gaithersburg) was connected to OSINET via a native $\mathbb{X} .25$ board and to an IEEE 802.3 LAN via a native controller board. ESE was connected to the IEEE 802.3 LAN via a native controller board. The ICL ISDN workstation's (ESB) connection to the 5ESS (TM) switch was the same as for Path 2. The software view was composed of an OSI stack containing FTAM, TP4, and CLNP.

The fifth path involved the sending of electronic mail between the Retix ES (ESD) and an ATßT Mail PMX/X.400 system (ESF). Each system was attached to the 5ESS (TM) switch via a BRI Terminal Adapter over preallocated B-Channels. The software for this test consisted of an OSI stack containing MHS and TPO directly over X.25 (i.e., using the Connection-mode Network Service).

A modification was made to the above scenario to include electronic mail sent from a remote ISDN terminal via the ATজT Mail PMX/X.400 system (ESF) to the Retix ES (ESD). The remote ISDN terminal was attached to an AT\&T System 85, which in turn communicated to the 5ESS (TM) switch via a Primary Rate Interface (PRI). From the 5ESS (TM) switch the connection to the AT\&T Mail PMX/X.400 system (ESF) and the Retix ES (ESD) were as described above. 


\subsection{The Trial Parameters}

In order to minimize interoperation problems, settings for most configurable parameters were agreed upon before the trial. Rather than listing the protocols in detail, extensive reference is made to Version 2, Edition 1 of the NIST/OSI Implementors' Workshop Stable Agreements (SAs) approved at the December, 1988 meeting of the NIST Workshop for Implementors of OSI. The suggested parameter settings are given below:

* I.430 Basic Rate Access: Refer to SAs.

* LAPD: Refer to SAs. The following parameters were agreed to: $k$ (window size) $=3$, maximum frame size $=263$ (i.e., allow for an X.25 DATA packet with a User Data Field size of 256 octets), and modulo 128 numbering.

* LAPB: Refer to SAs. The following parameters were specified: $k$ (window size) $=7$, maximum frame size $=263$, Tl retransmission timer $=1$ second, and $\mathrm{N} 2$ retransmission count $=3$.

* X.25 Packet Layer Protocol: The following parameters were decided upon: Window size $=3$, Packet $\mathrm{Size}=256$, modulo 8 numbering, Flow Control Parameter Negotiation Facility, use of SVC setup in either direction, and multiple logical channels. X.121 address strings were 14 digits or less.

* ISO 8473 CLNP: It was agreed not to use the segmenting/reassembly function of CLNP. It was also agreed not to use any Type 2 or 3 functions. OSI NSAP addresses were based on the format used for OSINET and a temporary ORG ID was assigned.

* ISO 9542 ES-IS: It was agreed not to use this protocol.

* IEEE 802.3 CSMA/CD: Refer to SAS.

* IEEE 802.2 LLC: It was agreed that Type 1 LLC would be used. The LSAP for identifying ISO 8473 was hex ' $F E$ '.

* ISO 8073 Transport: It was agreed that Class 4 would be used for both MHS and FTAM. It was also agreed to use a TPDU size of $1 \mathrm{~K}$ octets, to negotiate no use of checksums, to use a retransmission timer of 30 seconds, and a retry counter of 3 . Inactivity and Keep Alive functions would be used. No QOS nor user data would be carried in the CR or CC TPDUs. It was proposed that a null TSAP ID be used. 
* Session Layer: It was agreed to allow use of either Session version 1 or version 2 (one system was capable of supporting both versions).

\subsection{Additions to the Trial Configuration}

As mentioned above, there were 5 main communication paths specified for the trial. Two additional paths were added, paths 6 and 7 . These paths were not critical, but were "extra credit" paths to be tested if time permitted. The following paragraphs will give the details of these two added paths.

Path 6 was similar to path 4. Here instead of the ICL ISDN workstation (ESB) exchanging files with NIST's remote location (ESE) over OSINET, it exchanged files with General Motors over OSINET. The hardware configuration was almost identical to that of path 4 , with one exception. The IS and ES located at General Motors (IS4 and ESG) were connected via an IEEE 802.4 IAN, rather than an IEEE 802.3. The software stack was an OSI stack consisting of FTAM, Session Version 2, TP4, and CLNP.

Path 7 was identical to the first path, with one exception. In this test, ICL (ESB) exchanged files with its home location (ESH), the United Kingdom, over OSINET. This test involved utilizing an X.75 gateway between ATßT'S ACCUNET(R) Packet Service (the OSINET backbone in the US) and the United Kingdom's X.25 service provider. The OSI stack used was the same as that specified in the above path.

\subsection{The Trial Results}

The trial was held June $20 \& 21$, 1989, at Mather Air Force Base, California. Each of the above mentioned communication paths were tested. The results of testing are discussed in the following paragraphs noting only exceptions that arose. The results will include not only the outcome of the testing, but also any parameter changes (from those specified earlier), and any problems or reservations.

Testing of all of the paths went well. In the FTAM testing between the NIST OSI workstation (ESA) and the ICL ISDN workstation (ESB), path 2 , a parameter setting was changed. The maximum TPDU size was reset to a lower value; both the values 128 and 256 were used. This was necessary to enable file transfer operations because of the lack of segmenting and reassembly. Please note that a change of nature is common in interoperability testing. 
Initially, testing of paths 2 and 3 , could only be initiated in one direction, due to a software problem of recognizing and handling Call User Data. This problem was later resolved to allow testing to be intiated from both directions. It was felt that had more extensive testing been done prior to the trial, this problem would have been discovered and resolved earlier.

There was success in establishing a Transport connection over a MAP 802.4 network between the ICL ISDN workstation (ESB) and General Motors (ESG), path 6 . But, due to lack of time, significant file transfer was not done.

\subsection{The Trial Summary}

The following sections will review the ISDN capablilities proposed for inclusion in GOSIP Version 2 and comment on the ISDN functions demonstrated during the trial. The ISDN functions not tested will also be discussed.

\subsection{ISDN Functions Proposed in GOSIP Version 2}

As mentioned earlier, the main thrust of the trial was to demonstrate the use of ISDN as a lower layer technology for OSI applications. It was felt that the trial was a necessary step to ISDN being included in GOSIP Version 2. It is therefore important to know the exact ISDN capability proposed for GOSIP Version 2 . Listed below are the particular ISDN functions proposed for inclusion:

* Connectionless Network Protocol (CLNP) over ISDN X.25

* Optional Connection Oriented Network Service (CONS) over ISDN X.25

* ISDN D-ChanneI

* ISDN Dynamic B-Channel

* ISDN Preallocated B-Channel

* Basic Rate Interface (BRI)

* Primary Rate Interface (PRI)

* S, T, and U Interfaces

\subsection{ISDN Functions Demonstrated During The Trial}

Overall, the trial was a success. Below ISDN functions proposed for GOSIP Version 2 are contrasted with the functions demonstrated 
during the trial. The ISDN functions for GOSIP Version 2 are listed above. The ISDN functions tested during the trial and the capability they provided are discussed below.

About $70 \%$ of the ISDN functions proposed for inclusion in GOSIP Version 2 were demonstrated during the trial. Listed below are the ISDN functions that were demonstrated:

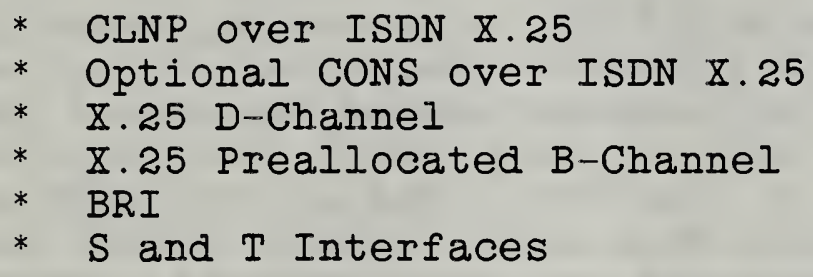

The above listed functions allowed demonstration of the following ISDN capabilities:

* ISDN as a transit subnetwork along an end-to-end communication path where neither the source nor the destination are directly attached to the ISDN itself and the transit properties of an ISDN as an OSI subnetwork and

* interconnectivity capability of an ISDN subnetwork with other types of subnetworks and end-to-end communication across multiple interconnected subnetworks where one of the subnetworks is an ISDN.

\subsection{ISDN Functions Not Demonstrated During The Trial}

There are several functions of ISDN which were not sufficiently tested during the trial. The use of X.25 over dynamic B-Channels was not tested because the trial planning indicated that computer vendors could not have interface boards ready by the target date (originally April). While such capabilities were available in June, the trial technical plan was already established. The second item which was not addressed is interoperability between ISDN Customer Premises Equipment (CPE) and a range of vendor switches (this is particularly sensitive to dynamic allocation of B-Channels). A third item which was not proven during the trial is interoperability between ISDN switches from a range of manufacturers. Items two and three were not proven since only one switch was utilized for the trial.

There are two observations important to note. First, of the above mentioned functions, the first two are GOSIP Version 2 related; 
the last item is not. Second, the ISDN functionality discussed above could have been tested had additional vendors participated in the trial and/or the trial had been scheduled later.

\subsection{Final Thoughts}

In terms of the GOSIP Version 2 ISDN functionality tested, the trial was successful. The slight problems experienced during testing are quite common to interoperability testing and testing of pre-release software. But, in the case of this trial, these problems were slight, and satisfactorily resolved. The use of ISDN as a lower layer technology would have been more strongly proven had the use of X.25 over dynamic B-Channels been demonstrated. However, that was not possible in this trial.

The most vulnerable area, and one of considerable concern, is the interoperability of Terminal Equipment (TE) and vendor switches. This is an area where more work must be done to assure users that equipment interoperates. Similar efforts are currently underway among the vendors of OSI applications. It is essential to users that interoperability concerns be addressed by ISDN vendors within the mechanisms established by the North American ISDN Users Forum (NIU Forum). 


\section{REFERENCES}

Burg, F.M., Di Iorio, N., Wilcock, W.T., "NIST OSI/ISDN Trial: Project Plan, Issue 2.2" April 28, 1989.

Burg, F.M., Di Iorio, N., Wilcock, W.T., "NIST OSI/ISDN Trial: Technical Prospectus, Issue 1.0" April 28, 1989.

FIPS 146, "Government Open Systems Interconnection Profile (GOSIP)", NTIS, U.S. Department of Commerce, 5285 Port Royal Road, Springfield, VA 22161.

NIST, "OSIone FTAM Interoperability Tests", Version 1.0, June 1989.

NIST, "OSINET/EurOSInet X.400 Interoperability Tests", Version 1.00 , June 1989.

Proposed FIPS, "Government Open Systems Interconnection Profile (GOSIP)", Version 2.0 Draft, April 1989.

Zawadzki, A., "NIST OSI/ISDN Trial: System Test Plan", May $11,1989$. 
APPENDIX A - List of Contacts

NAME

Fred Burg

COMPANY

ATET BL

Nick Di Iorio

Ned McCook

Howard Fingeroot

Ron Aitchison

Kevin Mills

Carol Edgar

Jeff Marx

Scott Kuklin

Howard Fiderer

Dave Brabender
DGM\&S

General Motors

ICL, Inc

NIST

Retix

Teleos (TM)

Communications

U.S. Air Force
ADDRESS \& TELEPHONE

Room 1M-325

Crawfords Corner Road

Holmdel, NJ 07733

(201) 949-0919

Room 1M-317

Crawfords Corner Road

Holmdel, NJ 07733

(201) 949-8741

1025 Briggs Road

Mt. Laurel, NJ 08054

(609) 866-1212

General Motors Tech. Ctr. 30300 Mound Road - BMD-39

Warren, MI 48090-9040 (313) 947-0564

777 Long Ridge Road P.O. Box 10276

Stamford, CT 06904 (203) $-968-7200$

Bldg. 225, Room B-217

Gaithersburgీ, MD 20899

(301) 975-3643

2644 30th Street

Santa Monica, Ca 90405

(213) 399-2200

2 Meridian Road

Eatontown, NJ 07724

(201) 389-5700

$323 \mathrm{FTW} / \mathrm{SC} 4$

Bldg. 3577

Mather AFB, CA 95655-5000

(916) 364-4433 
4. TITLE AND SUBTITLE

Trial of Open Systems Interconnection (OSI) Protocols over Integrated Services Digital Vetwork (ISDN)

5. AUTHOR(S)

Carol A. Edgar

6. PERFORMINO ORGANIZATION (IF JOINT OR OTHER THAN NIST, SEE INSTRUCTIONS)

U.S. DEPARTMENT OF COMMERCE

NATIONAL INSTITUTE OF STANDARDS AND TECHNOLOGY

GAITHERSBURG, MO 20899

9. SPONSORING ORGANIZATION NAMEAND COMPLETE ADORESS (STREET, CITY, STATE, ZIP)

\section{SUPPLEMENTARY NOTES}

DOCUMENT DESCRIBES A COMPUTER PROGRAM; SF-185, FIPS SOFTWARE SUMMARY, IS ATTACHED.

11. ABSTRACT (A 20O-WORO OR LESS FACTUAL SUMMARY OF MOST SIGNIFICANT INFORMATION. IF DOCUMENT INCLUDES A SIGNIFICANT BIBUOGRAPHY OR

This document contains the results of the NIST Open Systems Interconnection/ Integrated Services Digital Network (OSI/ISDN) Trial. The trial was organized to demonstrate the use of ISDN as a lower layer technology for oSI applications.

The document addresses the trial's topology, hardware/software configuration, parameters, and results.

12. KEY WOROS (6 TO 12 ENTRIES; ALPHABETICAL ORDER; CAPITAUZE ONLY PROPER NAMES; AND SEPARATE KEY WORDS BY SEMICOLONS) domonstration; connectivity; Government Open Systems Interconnection Profile; GOSIP Version 2; Integrated Services Digital Network; ISDN; interoperation; 10wer layer technology; NIST OSI/ISDN Trial; Open Systems Interconnection; OSI; transport technologies. 1

FOR OFFICIAL DISTRIBUTION. DO NOT RELEASE TO NATIONAL TECHNICAL INFORMATION SERVICE (NTIS).

ORDER FROM SUPERINTENDENT OF DOCUMENTS, U.S. GOVERNMENT PRINTING OFFICE, WASHINGTON, DC 20402.

ORDER FROM NATIONAL TECHMICAL INFORMATION SERVICE (NTIS), SPRIMGFIELD, VA 22161.

\begin{tabular}{|l|} 
14. NUMBER OF PRINTEO PAGES \\
20 \\
15. PRICE \\
\end{tabular}




